\title{
Red Wine Age Estimation by the Alteration of Its Color Parameters: Fourier Transform Infrared Spectroscopy as a Tool to Monitor Wine Maturation Time
}

\author{
M. Basalekou, ${ }^{1}$ C. Pappas, ${ }^{2}$ Y. Kotseridis, ${ }^{1}$ P. A. Tarantilis, ${ }^{2}$ \\ E. Kontaxakis, ${ }^{3}$ and S. Kallithraka ${ }^{1}$ \\ ${ }^{1}$ Laboratory of Oenology, Department of Food Science \& Human Nutrition, Agricultural University of Athens, 75 Iera Odos, \\ 11855 Athens, Greece \\ ${ }^{2}$ Laboratory of Chemistry, Department of Food Science \& Human Nutrition, Agricultural University of Athens, 75 Iera Odos, \\ 11855 Athens, Greece \\ ${ }^{3}$ Department of Agriculture, School of Agriculture, Food and Nutrition, Technological Educational Institute of Crete, \\ Estavromenos, 71004 Heraklion, Greece
}

Correspondence should be addressed to S. Kallithraka; stamatina@aua.gr

Received 28 April 2017; Revised 12 September 2017; Accepted 26 September 2017; Published 1 November 2017

Academic Editor: Larisa Lvova

Copyright (C) 2017 M. Basalekou et al. This is an open access article distributed under the Creative Commons Attribution License, which permits unrestricted use, distribution, and reproduction in any medium, provided the original work is properly cited.

Color, phenolic content, and chemical age values of red wines made from Cretan grape varieties (Kotsifali, Mandilari) were evaluated over nine months of maturation in different containers for two vintages. The wines differed greatly on their anthocyanin profiles. Mid-IR spectra were also recorded with the use of a Fourier Transform Infrared Spectrophotometer in ZnSe disk mode. Analysis of Variance was used to explore the parameter's dependency on time. Determination models were developed for the chemical age indexes using Partial Least Squares (PLS) (TQ Analyst software) considering the spectral region $1830-1500 \mathrm{~cm}^{-1}$. The correlation coefficients $(r)$ for chemical age index i were 0.86 for Kotsifali (Root Mean Square Error of Calibration $($ RMSEC) = 0.067, Root Mean Square Error of Prediction $($ RMSEP $)=0,115$, and Root Mean Square Error of Validation $($ RMSECV) $=0.164)$ and 0.90 for Mandilari (RMSEC $=0.050$, RMSEP $=0.040$, and RMSECV $=0.089$ ). For chemical age index ii the correlation coefficients $(r)$ were 0.86 and 0.97 for Kotsifali (RMSEC 0.044, RMSEP $=0.087$, and RMSECV $=0.214$ ) and Mandilari $(\mathrm{RMSEC}=0.024$, RMSEP $=0.033$, and RMSECV $=0.078)$, respectively. The proposed method is simpler, less time consuming, and more economical and does not require chemical reagents.

\section{Introduction}

Wine age is a parameter of great importance as it is linked with wine quality. However, there is no direct way to measure it. Most information related to age is derived from the evolution of the wine's organoleptic characteristics, such as color, mouth feel, and aroma which depend on the phenolic and volatile content of the wine.

Color in particular is an indicator of the interactions between phenolic compounds, such as the replacement of monomer forms by polymers which are time dependent reactions. Young wines' color depends on the concentration of free anthocyanins, which on the wine's $\mathrm{pH}$ are on their colored form (positively charged flavylium). As monomers, they are highly susceptible to changes in the medium's environment, such as $\mathrm{pH}$ and $\mathrm{SO}_{2}$ changes [1]. During ageing, while monomers are replaced by polymers, color changes and stabilization occur. For example, color density is reduced as a result of oxidation but also due to changes in the structure of anthocyanin-tannin polymers and precipitation of some of the polymers formed. Moreover, the purple hue of young red wines is replaced by brickish red to tawny red hues. Those changes in color can be observed with the naked eye, but also easily using a spectrophotometer by measuring color parameters such as intensity and hue [2]. The transformation of monomeric pigments to more stable polymeric 
forms is well documented in research [3,4]. The evolution of the physicochemical characteristics of wines occurs primarily during wine maturation and ageing, as phenolic compounds participate in numerous chemical reactions such as copigmentation and polymerization. One of them is the gradual formation of condensed pigments between free anthocyanins and colorless phenols present in grapes. These compounds can be formed either in the presence or in the absence of oxygen. When ageing is oxidative, that is, barrel ageing or vat ageing with high levels of aeration, the anthocyanins and tannins react also with acetaldehyde which is formed as a result of ethanol oxidation, to form pigments of various structures. These reactions are catalysed by ellagitannins, phenols that are extracted from the barrel [5]. The polymeric pigments can be further classified into two categories: long polymeric pigments (LPP) and short polymeric (SPP) pigments. LPP have a polymer length greater than three and can be precipitated with protein while SPP are shorter polymers that do not precipitate with proteins [6]. Copigmentation refers to the self-association of anthocyanins or their association with certain phenolic monomers and results in color stabilization due to hyperchromic and bathochromic shifts in the visible absorbance [7].

Monitoring the evolution of anthocyanins can be a demanding task in terms of analytical methods. For a time efficient and in-depth analysis of the anthocyanin forms over time, without the use of specified instruments, Somers introduced in 1977 the concept of "chemical age." Chemical age is defined as the extent to which monomeric anthocyanins have been displaced by polymeric pigment forms [8]. Indices of chemical age are the indexes "chemical age i" and "chemical age ii," which are derived from two spectral ratios. Both refer to the extent to which pigments have become less susceptible to the changes of $\mathrm{pH}$ and to $\mathrm{SO}_{2}$ bleaching and changes to which free anthocyanins are more susceptible as less stable. The sample absorbance at $520 \mathrm{~nm}$ is due to the free forms of anthocyanins, copigmented anthocyanins, and polymeric pigments. Absorbance is measured after $\mathrm{pH}$ adjustment or the addition of bisulfite solution or acetaldehyde to separate total anthocyanins and polymeric pigments. Lowering the $\mathrm{pH}$ of the wine allows the determination of free anthocyanins and polymeric pigments; acetaldehyde removes the bleaching effect of any $\mathrm{SO}_{2}$ present in the wine and the addition of bisulfite reveals the degree to which the color is due to the polymeric anthocyanin forms [9]. This UV-Vis based method commonly used in the wineries requires multiple step sample processing and measurements.

Given the importance of wine color, an indirect method able to determine the color parameters in red wines with reasonable accuracy and ease of use would be of great value to wine industry. Infrared spectroscopic methods have the advantage of being nondestructive and fast and require small sample quantities. When combined with multivariate data analysis, such as Partial Least Squares (PLS) regression analysis, they are suitable for correlating the spectral response of a sample with its chemical composition [10]. The application of both near- and mid-infrared spectroscopic methods to grape and wine analysis has become a valuable alternative to the slow and destructive analytical procedures which are routinely used as the classic UV-Vis and chromatographic techniques [11-16] or even for monitoring wine ageing in barrels [17].

The first applications of FT-IR for the quantification of total anthocyanins in wine were carried out by Versari et al. [18]. They also determined LPP, SPP, total wine color, and copigmented anthocyanins. The best results were obtained using vector normalization and no-preprocessing of spectral data. Later studies using direct orthogonal signal correction preprocessing have improved the prediction of polymeric pigments in red wines by FT-IR spectroscopy compared to the row data [19]. Picque et al. [20] also used FT-IR spectroscopy to build a predictive model that was able to quantify anthocyanins in red grape extracts. Anthocyanin prediction was improved if a separate calibration model was calculated for each geographical region. The best prediction for monitoring anthocyanins in red grapes using FT-IR was presented by Fragoso et al. [12]. They obtained a valid regression model for prediction of total anthocyanins working in the region of $979-2989 \mathrm{~cm}^{-1}$.

Individual anthocyanin concentration can also be predicted using FT-IR [21] in young red wines. Recently, RasinesPerea et al. [22] determined twelve individual anthocyanins in red grape musts using FT-IR and PLS. However, in both the above studies there was a need to employ correction factors to improve the prediction due to systematic errors.

There exist a number of important studies concerning the changes in phenolic content and color parameters of red wines during ageing [23-25]. However, despite the importance of visual appearance to the final wine quality, there is not much published data concerning the correlation of color parameters with wine age. It was thus of interest to determine the alteration of selected color parameters of red wines during short maturation periods and to evaluate the overall changes with respect to their age.

Moreover, it was of interest to explore the suitability of FTIR spectroscopy as a simple, less time consuming, and more economical technique to monitor wine chemical age which is of high technological importance.

\section{Materials and Methods}

2.1. Wines and Containers. The wines used were made of two major red grape varieties of Crete, Kotsifali and Mandilari, which differ greatly in their anthocyanic and tannic content. Kotsifali produces wines low in color and relatively high in alcohol with smooth tannins, whereas Mandilari produces age worthy wines with deep red color. All wines were vinified following the protocol for classical red winemaking and received the same $\mathrm{SO}_{2}$ additions. No tartaric acid additions were made as $\mathrm{pH}$ values for all wines were satisfactory ( $\mathrm{pH}$ 3.4-3.5). For the maturation process, different types of containers were used (tank, tank with French oak chips and barrels made of French oak, American oak, Acacia, and Chestnut) and samples were taken from each container every three months over the period of nine months for two consecutive vintages (2012 and 2013) (12 months of contact only for the 2013 vintage), resulting in 12 red wine samples for each vintage's trimester (Table 1). 
TABLE 1: Samples and containers per vintage (V1: 2012; V2: 2013).

\begin{tabular}{|c|c|c|c|c|c|}
\hline \multirow{2}{*}{ Grape variety } & \multirow{2}{*}{ Container } & \multicolumn{4}{|c|}{ Samples } \\
\hline & & 3 months & 6 months & 9 months & 12 months \\
\hline \multirow{6}{*}{ Kotsifali/Mandilari } & Inox tank & $\mathrm{V} 1 / \mathrm{V} 2$ & $\mathrm{~V} 1 / \mathrm{V} 2$ & $\mathrm{~V} 1 / \mathrm{V} 2$ & $\mathrm{~V} 1 /-$ \\
\hline & Inox tank with oak chips & $\mathrm{V} 1 / \mathrm{V} 2$ & $\mathrm{~V} 1 / \mathrm{V} 2$ & $\mathrm{~V} 1 / \mathrm{V} 2$ & $\mathrm{~V} 1 /-$ \\
\hline & French oak & $\mathrm{V} 1 / \mathrm{V} 2$ & $\mathrm{~V} 1 / \mathrm{V} 2$ & $\mathrm{~V} 1 / \mathrm{V} 2$ & $\mathrm{~V} 1 /-$ \\
\hline & American oak & $\mathrm{V} 1 / \mathrm{V} 2$ & $\mathrm{~V} 1 / \mathrm{V} 2$ & $\mathrm{~V} 1 / \mathrm{V} 2$ & $\mathrm{~V} 1 /-$ \\
\hline & Acacia & $\mathrm{V} 1 / \mathrm{V} 2$ & $\mathrm{~V} 1 / \mathrm{V} 2$ & $\mathrm{~V} 1 / \mathrm{V} 2$ & $\mathrm{~V} 1 /-$ \\
\hline & Chestnut & $\mathrm{V} 1 / \mathrm{V} 2$ & $\mathrm{~V} 1 / \mathrm{V} 2$ & $\mathrm{~V} 1 / \mathrm{V} 2$ & $\mathrm{~V} 1 /-$ \\
\hline \multicolumn{2}{|c|}{ Sum of samples for both varieties } & $12 / 12$ & $12 / 12$ & $12 / 12$ & $12 /-$ \\
\hline
\end{tabular}

2.2. Spectrophotometric Analyses. Total anthocyanins (TA), degree of ionisation of anthocyanins (ID), color density (CD), color density corrected for $\mathrm{SO}_{2}$ (CDS), $\mathrm{SO}_{2}$ resistant pigments (SRP), and chemical ages $\mathrm{i}$ and ii were determined according to the modified Somers assay as described by Mercurio et al. [1]. According to the method, prior to analysis $\mathrm{pH}$ and alcohol content of all wine samples were standardized to 3.4 and $12 \% \mathrm{v} / \mathrm{v}$, respectively, with the use of a buffer solution. After that, the wines are treated with excess $\mathrm{SO}_{2}$, excess acetaldehyde, and hydrochloric acid. The absorbance of the samples is read in four steps: first, the absorbance of wine in its original state is read at 420 and $520 \mathrm{~nm}$ ( $A_{420 \text { buffer }}, A_{520 \text { buffer }}$ ); then the second reading is after the addition of excess SO2 $\left(A_{520}\right.$ sulfite $)$. This reading allows the measurement of color resulting from SO2-resistant pigments. Subsequently, the absorbance of wine samples treated with excess acetaldehyde is read $\left(A_{520 \text { acetal }}\right)$, to estimate the anthocyanins which are colored at wine $\mathrm{pH}$. Finally, the absorbance of wine diluted with hydrochloric acid is read $\left(A_{520 \mathrm{HCl}}\right)$. This treatment lowers the $\mathrm{pH}$ of wine and this way all anthocyanins are converted into their colored forms.

\section{Calculations}

Total anthocyanins $(\mathrm{mg} / \mathrm{L}): 20 \times\left[\left(50 \times A_{520 \mathrm{HCl}}\right)-\right.$ $\left.\left(1.6667 \times\left(10 \times A_{520 \text { sulfite }}\right)\right]\right)$.

Degree of ionisation of anthocyanins (\%): $100 \times[(10 \times$ $\left.\left.A_{520 \text { buffer }}\right)-\left(10 \times A_{520 \text { sulfite }}\right)\right] /\left[\left(50 \times A_{520 \mathrm{HCL}}\right)-\right.$ $\left.1.6667 \times\left(10 \times A_{520 \text { sulfite }}\right)\right]$.

Color density (au): $\left(A_{420 \text { buffer }}+A_{520 \text { buffer }}\right) \times 10$.

Color density corrected for $\mathrm{SO}_{2}(\mathrm{au}):\left(A_{420 \text { acetal }}+\right.$ $\left.A_{520 \text { acetal }}\right) \times 10$.

$\mathrm{SO}_{2}$ resistant pigments (au): $A_{520 \text { sulfite }} \times 10$.

Chemical age i (no units): $A_{520}$ sulfite $/ A_{520 \text { acetal }}$.

Chemical age ii (no units): $A_{520 \text { sulfite }} /\left(5 \times A_{520 \mathrm{HCl}}\right)$.

Both indexes are close to zero in wines right after fermentation is completed [8].

Color intensity (CI) and hue (h) were determined according to the method proposed by Glories [26] and were calculated after measuring the absorbance at 420, 520, and $620 \mathrm{~nm}$ as follows: $\mathrm{CI}=A_{420}+A_{520}+A_{620}$ and $\mathrm{h}=A_{420} / A_{520}$.

All absorbance measurements were performed on a Hitachi U-2000 spectrophotometer.
2.3. FT-IR. Mid-IR spectra of all samples were collected with the use of a Fourier Transform Infrared Spectrophotometer (Thermo Nicolet 6700 FT-IR by Thermo Electron Corporation, MA, USA) equipped with a deuterated triglycine sulfate (DTGS) detector, following the procedure described by Basalekou et al. [17]. All samples' spectra were recorded in the region $4000-500 \mathrm{~cm}^{-1}$.

2.4. Statistical Analysis. Principal Component Analysis (PCA) and Analysis of Variance (ANOVA) were performed to plot differences between samples and to examine each variable's (vintage, time, and container) effect on wine color compounds. The statistical program used for these analyses was JMP v.11. Determination models were also developed using Partial Least Squares (PLS) and the FT-IR's built-in software TQ Analyst. PLS is a quantitative analysis technique. The PLS algorithm examines the specified region of the calibration spectra to determine areas that vary statistically as a function of component concentration. The calibration model is then developed using spectral and concentration information. The spectral region considered for the statistical analysis was $1830-1500 \mathrm{~cm}^{-1}$.

\section{Results and Discussion}

3.1. Chemical Analyses. Chemical analyses highlighted the differences in the phenolic content of Kotsifali and Mandilari wines. For Kotsifali, the maximum initial average concentration of anthocyanins (i.e., after fermentation completion) was $145.4 \mathrm{mg} / \mathrm{L}$ while for Mandilari it was $322.6 \mathrm{mg} / \mathrm{L} \mathrm{g} / \mathrm{L}$ (vintage, 2013). Mandilari wines are characterized as being rich in anthocyanins and tannins, presenting a deep red color and a quite astringent palate. Their high tannic and anthocyanin concentration favours the formation of more anthocyanin-tannin polymers.

The anthocyanin concentration of Kotsifali and Mandilari wines decreased threefold over the nine-month period of maturation for both vintages and in all containers (Table 2). Indeed, anthocyanins are unstable molecules and they are incorporated into the tannin structure, forming pigmented polymers. The decrease in anthocyanin concentrations is also associated with the formation of more stable pigments such as pyranoanthocyanins as well as the degradation of anthocyanins [27]. These results are in agreement with the results obtained by Kallithraka et al. [28]. The method used 
TABLE 2: Changes in average values of color parameters and chemical age indexes (CI: color intensity, h: hue, CA1: chemical age i, CA2: chemical age ii, ID: degree of ionisation of anthocyanins, CD: color density, CDS: color density corrected for $\mathrm{SO}_{2}$, TA: total anthocyanin concentration, SRP: $\mathrm{SO}_{2}$ resistant pigments) during maturation, for Kotsifali and Mandilari wines.

\begin{tabular}{|c|c|c|c|c|c|c|}
\hline & & 2012 vintag & & & 2013 vintag & \\
\hline & 3 months & 6 months & 9 months & 3 months & 6 months & 9 months \\
\hline Kotsif & & & & & & \\
\hline CI & 2.8 & 3.4 & 3.9 & 5.5 & 5.1 & 6.1 \\
\hline $\mathrm{h}$ & 0.9 & 0.9 & 1.0 & 0.7 & 0.8 & 0.8 \\
\hline CA 1 & 0.2 & 0.4 & 0.5 & 0.3 & 0.4 & 0.5 \\
\hline CA 2 & 0.1 & 0.1 & 0.2 & 0.1 & 0.1 & 0.2 \\
\hline ID & 18.5 & 15.5 & 28.3 & 23.7 & 23.0 & 24.8 \\
\hline $\mathrm{CD}$ & 2.9 & 4.0 & 3.2 & 4.4 & 4.4 & 4.5 \\
\hline CDS & 3.4 & 4.5 & 3.3 & 4.9 & 4.5 & 4.7 \\
\hline TA & 143.0 & 140.5 & 56.6 & 145.4 & 130.7 & 100.3 \\
\hline SRP & 0.4 & 1.1 & 0.9 & 1.0 & 1.1 & 1.3 \\
\hline Mand & & & & & & \\
\hline $\mathrm{CI}$ & 14.8 & 16.4 & 16.2 & 14.8 & 12.7 & 14.0 \\
\hline $\mathrm{h}$ & 0.5 & 0.5 & 0.5 & 0.5 & 0.5 & 0.6 \\
\hline CA 1 & 0.4 & 0.6 & 0.7 & 0.3 & 0.4 & 0.5 \\
\hline CA 2 & 0.2 & 0.3 & 0.4 & 0.1 & 0.2 & 0.2 \\
\hline ID & 32.8 & 34.4 & 57.4 & 28.1 & 26.8 & 27.7 \\
\hline $\mathrm{CD}$ & 11.2 & 13.1 & 11.6 & 10.6 & 10.1 & 9.9 \\
\hline CDS & 12.1 & 13.3 & 11.9 & 11.1 & 10.2 & 10.3 \\
\hline TA & 285.9 & 187.8 & 92.4 & 322.6 & 278.4 & 221.5 \\
\hline SRP & 3.2 & 5.2 & 4.9 & 2.6 & 2.9 & 3.3 \\
\hline
\end{tabular}

for anthocyanin determination is based on the effect of $\mathrm{pH}$ on anthocyanin structure. Considering that oligomeric and polymeric pigments are more resistant to $\mathrm{pH}$ changes than the monomeric ones [29] anthocyanin determination is based mainly on free monomeric anthocyanins. Therefore, the decrease observed in total anthocyanin content during maturation is consistent with the participation of monomeric anthocyanins in numerous condensation reactions as well as in hydrolytic and other degradation reactions [30] to a minor extent.

In general, all color parameters were characterized by higher values for Mandilari wines, given their higher anthocyanin content. Chemical age indexes $i$ and ii of Mandilari wines were also higher than the corresponding values measured in Kotsifali, indicating that in Mandilari wines more polymeric pigments are present at wine $\mathrm{pH}$ and that those polymeric forms are resistant to $\mathrm{SO}_{2}$ bleaching [31].

3.2. Statistical Analysis. To examine internal structure and patterns of the wine data set Principal Components Analysis was employed. The variables used, namely, total anthocyanins, degree of ionisation of anthocyanins, color density, color density corrected for $\mathrm{SO}_{2}, \mathrm{SO}_{2}$ resistant pigments, chemical ages $\mathrm{i}$ and ii, color intensity, and hue, produced the PCA plot shown in Figure 1.

The first two principal components explain $93.7 \%$ of the variance. As can be seen in Figure 1, the PCA plot resulted in a clear separation of the two varieties, based on the first principal component. However, a discrimination trend according to maturation time can also be observed, based on the second principal component. Most of the samples that have been aged for 3 months are situated lower than the rest of the samples that have been matured for 6 and 9 months, respectively. Moreover, samples that have been matured for 9 months are mostly found on the top of the diagram. As expected, this trend is more obvious between samples that aged for three months (lower part of the plot) and samples that aged for nine months (higher part of the plot) since it requires more than three months to observe differences in wine chemical parameters.

Given the strong influence of the variety on phenolic content and wine color which is evident in the PCA plot, Analysis of Variance was also performed to examine if any of the variables were independent of variety, vintage, and container effects and were only influenced by time. The results showed that total anthocyanins, hue, pigments resistant to $\mathrm{SO}_{2}$, and chemical ages $\mathrm{i}$ and ii were influenced by time; however, variety and vintage had a stronger effect in all cases except for chemical age i (Table 3) where time had the most definitive effect.

Table 3 presents the effect tests' results. The Nparm value indicates the number of parameters associated with the effect, DF is the degree of freedom, Sum of Squares gives the Sum of Squares for the hypothesis that the effect is zero, and $F$ ratio indicates whether the model differs significantly from a model where all predicted values are the response mean, while the Prob $>F$ value measures the probability of obtaining an $F$ ratio as large as what is observed. According to 


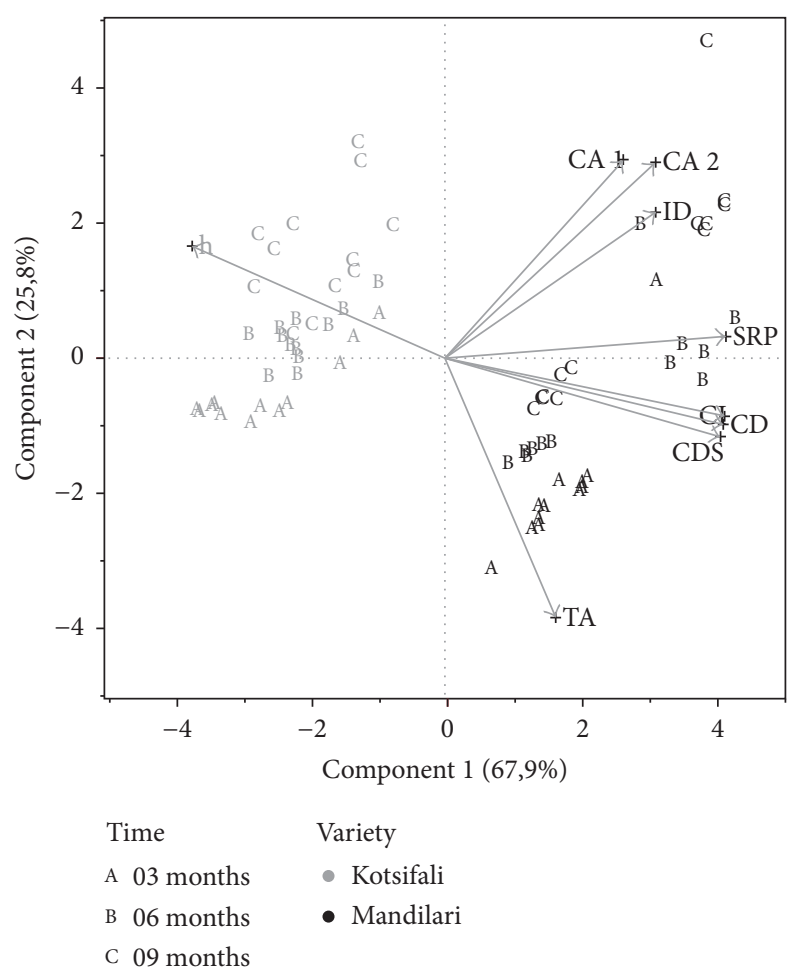

FIGURE 1: Biplot of principal components 1 and 2 for mean scores of Kotsifali and Mandilari color parameters.

Table 2, only the type of container does not have a statistically significant effect on the parameters measured, while the variety exhibits the strongest influence. Variety exhibits the strongest influence on hue ( $F$ ratio 617.9) followed by $\mathrm{SO}_{2}$ resistant pigments ( $F$ ratio 317.4) and total anthocyanin concentration ( $F$ ratio 121.1). Vintage seems to have a lesser effect, although in some cases it is more important than time. The only case where time is less dependent on variety and vintage is in chemical age index i (time $F$ ratio $61.3>$ variety $F$ ratio $30.1>$ vintage $F$ ratio 9 ).

3.3. FT-IR Analysis. According to Analysis of Variance, chemical age index $\mathrm{i}$ is the only parameter that is mostly time dependent and can produce significantly statistical differences in the short time period of nine months without strong variety interferences. The conventional analysis employed to measure chemical age indexes $\mathrm{i}$ and ii is simple but time consuming and needs chemical agents, making it less appealing to be implemented in the wineries as a routine protocol. When grape, must, or wine is analyzed for quality control, analysis time becomes a key parameter. For this purpose the suitability of FT-IR combined with PLS was investigated as an alternative method to provide information of such technological interest to the wineries.

For this reason, FT-IR coupled with chemometrics was used to develop calibration models. A typical FT-IR spectrum for Kotsifali and Mandilari samples is shown in Figure 2. The region that was selected for further statistical analysis was the region from 1830 to $1500 \mathrm{~cm}^{-1}$.

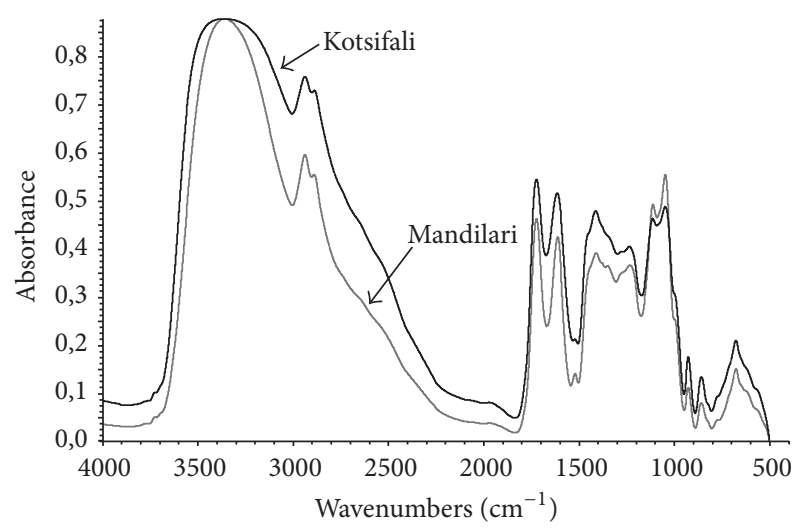

Figure 2: Typical FT-IR spectra for Kotsifali and Mandilari wines.

This area as part of the fingerprint region contains much information such as various IR-bands, including those corresponding to the vibrations of the $\mathrm{C}-\mathrm{O}, \mathrm{C}-\mathrm{C}$ and $\mathrm{C}-\mathrm{H}$ bonds [32]. As it is shown in Figure 2, two major peaks are included in the selected area. One centred at $1722 \mathrm{~cm}^{-1}$, due to the stretching of carbonyl group $(\mathrm{C}=\mathrm{O})$, and one at $1610 \mathrm{~cm}^{-1}$, due to $\mathrm{C}=\mathrm{C}$ stretching (typical for aromatic molecules) [33]. The first peak is mainly connected with the $\mathrm{C}-\mathrm{O}$ stretching of the esters of hydrolysable tannins, especially derivatives of gallic acid [34] and the $\mathrm{C}=\mathrm{C}$ stretching, typical of flavanoidbased compounds in wine [35], while the second is mainly attributed to the carboxyl ion (COO-) (symmetrical stretching) $[36,37]$. A smaller peak is also detected at $1520 \mathrm{~cm}^{-1}$, due to the deformation of the aromatic ring, which can be attributed to simple catechin [11,35]. The region from 1535 to $1520 \mathrm{~cm}^{-1}$ is affected by the structural modifications that occur during polymerization [35] and for this reason it was included in the spectral region used for the statistical analysis. Overall, the selection of the spectral region was based on absorptions that are due to the most important chemical compounds that participate in color stabilization reactions, such as phenolic acids, oak tannins, and flavonoids [7].

Wine spectra are extremely multivariate and hence complex. It is necessary to use advanced mathematical techniques to generate the calibration equations for the individual parameters. This type of calculations is often referred to as chemometrics. The multivariate regression methods have been widely used to provide a better insight into such systems and to build calibration and prediction models. Partial Least Squares (PLS) regression is one of the most used models [38] where the regression algorithm uses the absorptions at selected frequencies or blocks of frequencies to generate an equation that best fits the reference values in a data set. In wine analysis, those models were successfully applied for the determination of various compounds such as anthocyanins and tannins [11, 21], but also ethanol and organic acids (Moreira and Santos, 2005) [39].

In this experiment Partial Least Squares were used to develop calibration models for the chemical age indexes. The accuracy of the models is determined by their correlation coefficient $(r)$ and the Root Mean Square Error 
TABLE 3: Test results for fixed effects of hue, chemical age indexes i and ii, total anthocyanins, and $\mathrm{SO}_{2}$ resistant pigments for Kotsifali and Mandilari wine samples.

\begin{tabular}{|c|c|c|c|c|c|}
\hline & Nparm & $\mathrm{DF}$ & Sum of Squares & $F$ ratio & Prob $>F$ \\
\hline \multicolumn{6}{|l|}{ Hue } \\
\hline Variety & 1 & 1 & 2.081079 & 617.9852 & $<.0001^{*}$ \\
\hline Vintage & 1 & 1 & 0.0592846 & 17.6048 & $<.0001^{*}$ \\
\hline Time & 2 & 2 & 0.1038602 & 15.4209 & $<.0001^{*}$ \\
\hline Container & 5 & 5 & 0.0467252 & 2.775 & $0.0252^{*}$ \\
\hline \multicolumn{6}{|c|}{ Chemical age $i$} \\
\hline Variety & 1 & 1 & 0.15791902 & 30.0835 & $<.0001^{*}$ \\
\hline Vintage & 1 & 1 & 0.04734925 & 9.02 & $0.0038^{*}$ \\
\hline Time & 2 & 2 & 0.64369316 & 61.3116 & $<.0001^{*}$ \\
\hline Container & 5 & 5 & 0.04057336 & 1.5458 & 0.1888 \\
\hline \multicolumn{6}{|c|}{ Chemical age ii } \\
\hline Variety & 1 & 1 & 0.13732412 & 40.686 & $<.0001^{*}$ \\
\hline Vintage & 1 & 1 & 0.05456823 & 16.1673 & $0.0002^{*}$ \\
\hline Time & 2 & 2 & 0.20179451 & 29.8935 & $<.0001^{*}$ \\
\hline Container & 5 & 5 & 0.02202906 & 1.3053 & 0.2734 \\
\hline \multicolumn{6}{|c|}{ Total anthocyanins } \\
\hline Variety & 1 & 1 & 225840.64 & 121.065 & $<.0001^{*}$ \\
\hline Vintage & 1 & 1 & 42884.5 & 22.9888 & $<.0001^{*}$ \\
\hline Time & 2 & 2 & 139047.49 & 37.2692 & $<.0001^{*}$ \\
\hline Container & 5 & 5 & 8431.81 & 0.904 & 0.4843 \\
\hline \multicolumn{6}{|c|}{$\mathrm{SO}_{2}$ resistant pigments } \\
\hline Variety & 1 & 1 & 135.1368 & 317.3832 & $<.0001^{*}$ \\
\hline Vintage & 1 & 1 & 6.22457 & 14.6191 & $0.0003^{*}$ \\
\hline Time & 2 & 2 & 10.32205 & 12.1212 & $<.0001^{*}$ \\
\hline Container & 5 & 5 & 0.35078 & 0.1648 & 0.9745 \\
\hline
\end{tabular}

${ }^{*}$ indicates significant levels.

of Calibration (RMSEC) and Root Mean Square Error of Prediction (RMSEP) values. For Kotsifali wines, the models' correlation coefficient $(r)$ for chemical age i was 0.86 and the Root Mean Square Error of Calibration (RMSEC) and Root Mean Square Error of Prediction (RMSEP) were 0.066 and 0.115 , respectively (Figure $3(\mathrm{a})$ ). For Mandilari samples (Figure 3(b)), the correlation coefficient $(r)$ for chemical age $\mathrm{i}$ was $0.90(\mathrm{RMSEC}=0.050, \mathrm{RMSEP}=0.040)$. For each variety 42 samples were used to build the model, 9 of which were used for validation purposes. For Kotsifali 10 factors were used for each component calculated (Root Mean Square Error of Cross Validation $(\mathrm{RMSECV})=0.164)$, while for Mandilari 6 were used (RMSECV $=0.089$ ).

For chemical age ii the correlation coefficients $(r)$ for Kotsifali and Mandilari wine samples (Figures 4(a) and 4(b)) were 0.86 and 0.97 , respectively (Kotsifali: $\mathrm{RMSEC}=0.044$, RMSEP $=0.088$; Mandilari RMSEC $=0.024$, RMSEP $=0.033$ ). For each variety 42 samples were used to build the model, 9 of which were used for validation purposes. For both Kotsifali and Mandilari 10 factors were used for each component calculated (Kotsifali RMSECV $=0.214$, Mandilari RMSECV $=0.078$ ). Calibration and prediction values for all models as well as Predicted Residual Error Sum of Squares (PRESS) are given as Supplementary Material (Tables 1-4), available online at https://doi.org/10.1155/2017/5767613.
An interesting observation was that, in all cases studied, $r$ values of Mandilari were higher than the corresponding values of Kotsifali samples indicating a better fit of the proposed model for wines richer in phenolic compounds including anthocyanins. FT-IR is an indirect analytical method that is product sensitive. Every chemical substance has an IR "fingerprint" that is a function of the molecular bonds that are present in the sample. These characteristic signals are the basis of the measurement and are used in the calibration process. Different grape varieties may contain different compounds with similar IR absorption bands that may interfere with and disturb the calibration model. Indeed, the calibration models developed by FT-IR for musts or wines richer in anthocyanins and acids were more robust compared with those obtained for samples where the concentration of the compounds of interest was lower $[13,22]$.

In this study, $r$ was greater than 0.86 in all cases (higher than 0.90 in Mandilari wines), which is a satisfactory statistical significant value. The closer this value to "one" is, the more linear the relationship between the calculated and actual values is. RMSEC refers to the uncertainty of calibration while RMSEP estimates how well the method should predict concentration values for unknown samples. The calculated RMSEP values for all models were below 0.1, indicating low uncertainty values concerning the methods' prediction 

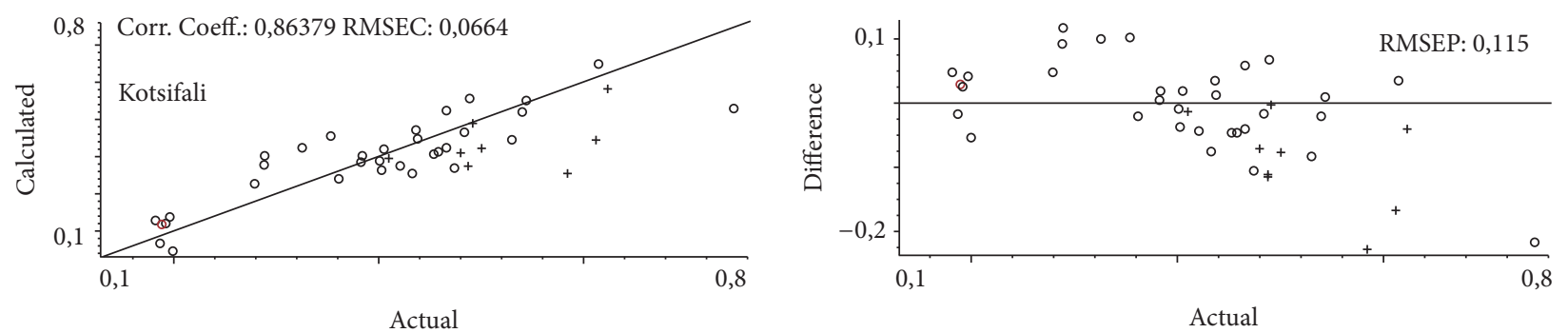

- Calibration

- Calibration

+ Validation

+ Validation

(a)
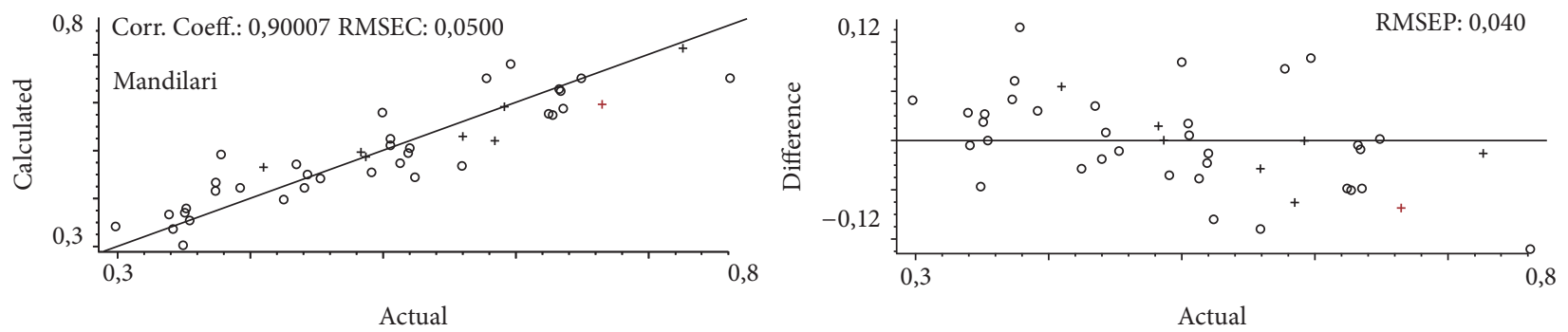

- Calibration

- Calibration

+ Validation

+ Validation

(b)

Figure 3: Correlation between the actual chemical age i values and the values predicted by the model for Kotsifali (a) and Mandilari (b) samples.
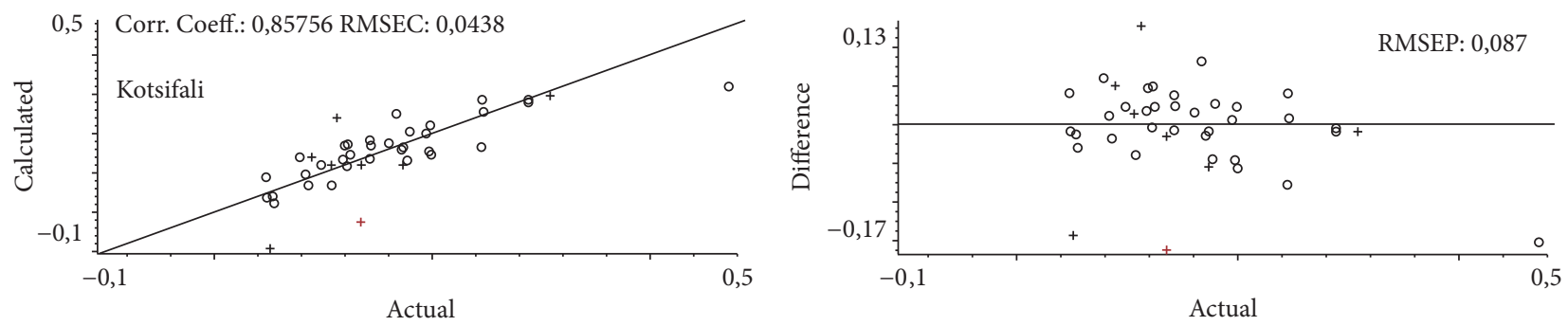

- Calibration

- Calibration

+ Validation

+ Validation

(a)
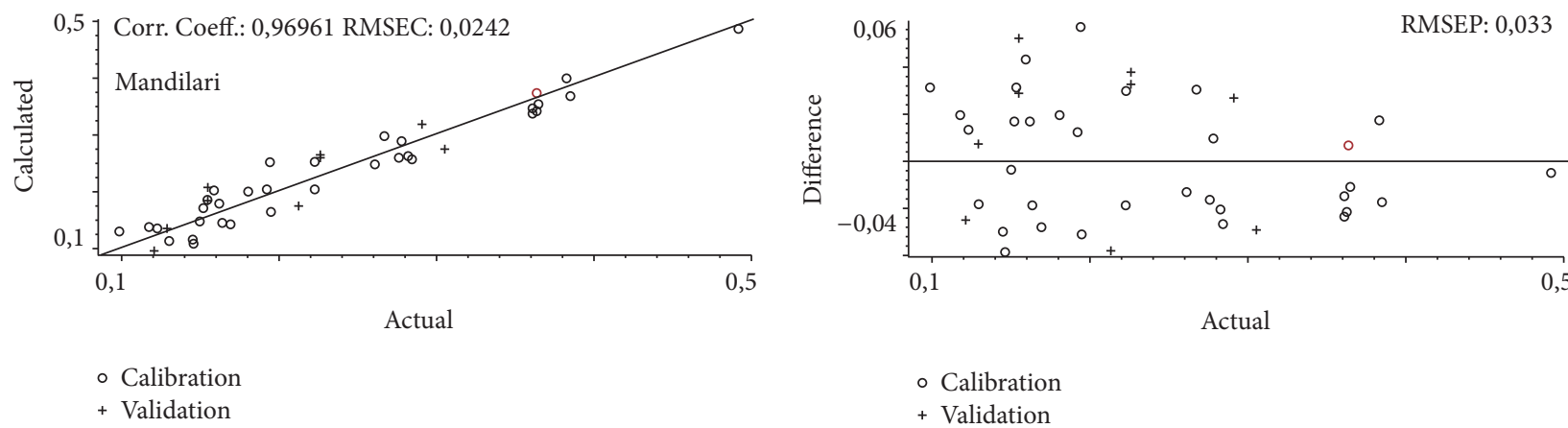

(b)

FIgURE 4: Correlation between the actual chemical age ii values and the values predicted by the model for Kotsifali (a) and Mandilari (b) samples. 
ability. The low values of RMSECV also demonstrate that the validation efficiency of the model is satisfactory. These data suggest that chemical age indexes may be estimated using FTIR and the aforementioned models.

As monomeric anthocyanins decrease during ageing color is no longer determined by monomeric forms; hence there is a lesser need to estimate them individually. In that case polymer forms are of greater importance, for the estimation of which chemical age indexes are more useful. To our knowledge this is the first time FT-IR is used to estimate the chemical age status of a wine.

\section{Conclusion}

Wine ageing has a defining effect on the color of red wines. Chemical age index i values are significantly correlated with maturation time and are less dependent on the variety, the vintage, or the type of container used for maturation. FTIR combined with PLS allowed developing regression models to provide approximate quantitative values for chemical age indexes in a quick and simple way that can be easily implemented for routine control of wines. Linear relationships were found with correlation coefficients $(r) 0.93$ and 0.91 for the chemical age index $i$ and 0.95 and 0.88 for index ii for Mandilari and Kotsifali samples, respectively. The low RMSEC values in each model demonstrate the robustness of the proposed method. It could be a starting point for the design of more specific models according to the requirements of the wineries.

\section{Conflicts of Interest}

The authors declare that there are no conflicts of interest regarding the publication of this paper.

\section{Acknowledgments}

This study was funded by the Thalis program, "Evaluation and Optimization of the Quality Factors during Maturation of Wines Produced from Cretan Red and White Grape Varieties: Production of High Quality Wines." This funding does not lead to any conflicts of interest regarding the publication of this manuscript.

\section{References}

[1] M. D. Mercurio, R. G. Dambergs, M. J. Herderich, and P. A. Smith, "High throughput analysis of red wine and grape phenolics-adaptation and validation of methyl cellulose precipitable tannin assay and modified somers color assay to a rapid 96 well plate format," Journal of Agricultural and Food Chemistry, vol. 55, no. 12, pp. 4651-4657, 2007.

[2] R. S. Jackson, Wine Tasting: A Professional Handbook, vol. 15, Food Quality and Preference, 3rd edition, 2017.

[3] M. Del Alamo Sanza, I. Nevares Domínguez, L. M. Cárcel Cárcel, and L. Navas Gracia, "Analysis for low molecular weight phenolic compounds in a red wine aged in oak chips," Analytica Chimica Acta, vol. 513, no. 1, pp. 229-237, 2004.

[4] E. Gómez-Plaza and M. Cano-López, "A review on micro-oxygenation of red wines: Claims, benefits and the underlying chemistry," Food Chemistry, vol. 125, no. 4, pp. 1131-1140, 2011.

[5] P. Ribéreau-Gayon, Y. Glories, A. Maujean, and D. Dubourdieu, Handbook of Enology, The Chemistry of Wine: Stabilization and Treatments, 2nd edition, 2006.

[6] J. F. Harbertson, E. A. Picciotto, and D. O. Adams, "Measurement of polymeric pigments in grape berry extracts and wines using a protein precipitation assay combined with bisulfite bleaching," American Journal of Enology and Viticulture, vol. 54, no. 4, pp. 301-306, 2003.

[7] R. Boulton, "The copigmentation of anthocyanins and its role in the color of red wine: A critical review," American Journal of Enology and Viticulture, vol. 52, no. 2, pp. 67-87, 2001.

[8] T. C. Somers and M. E. Evans, "Spectral evaluation of young red wines: Anthocyanin equilibria, total phenolics, free and molecular SO2, "chemical age"," Journal of the Science of Food and Agriculture, vol. 28, no. 3, pp. 279-287, 1977.

[9] T. C. Somers and E. Vérette, "Phenolic composition of natural wine types," in Wine Analysis, H. F. Linskens and J. E. Jackson, Eds., vol. 6 of Modern Methods of Plant Analysis, pp. 219-257, Springer, New York, NY, USA, 1988.

[10] A. Versari, G. P. Parpinello, and L. Laghi, "Application of infrared spectroscopy for the prediction of color components of red wines," Spectroscopy, vol. 27, no. 2, pp. 36-47, 2012.

[11] K. Fernández and E. Agosin, "Quantitative analysis of red wine tannins using Fourier-transform mid-infrared spectrometry," Journal of Agricultural and Food Chemistry, vol. 55, no. 18, pp. 7294-7300, 2007.

[12] S. Fragoso, L. Aceña, J. Guasch, M. Mestres, and O. Busto, "Quantification of phenolic compounds during red winemaking using FT-MIR spectroscopy and PLS-regression," Journal of Agricultural and Food Chemistry, vol. 59, no. 20, pp. 1079510802, 2011.

[13] U. Regmi, M. Palma, and C. G. Barroso, "Direct determination of organic acids in wine and wine-derived products by Fourier transform infrared (FT-IR) spectroscopy and chemometric techniques," Analytica Chimica Acta, vol. 732, pp. 137-144, 2012.

[14] S. D. Silva, R. P. Feliciano, L. V. Boas, and M. R. Bronze, "Application of FTIR-ATR to Moscatel dessert wines for prediction of total phenolic and flavonoid contents and antioxidant capacity," Food Chemistry, vol. 150, pp. 489-493, 2014.

[15] D. Cozzolino, "The role of visible and infrared spectroscopy combined with chemometrics to measure phenolic compounds in grape and wine samples," Molecules, vol. 20, no. 1, pp. 726-737, 2015.

[16] M. Kyraleou, C. Pappas, E. Voskidi et al., "Diffuse reflectance Fourier transform infrared spectroscopy for simultaneous quantification of total phenolics and condensed tannins contained in grape seeds," Industrial Crops and Products, vol. 74, pp. 784-791, 2015.

[17] M. Basalekou, C. Pappas, Y. Kotseridis et al., "Monitoring wine aging with Fourier transform infrared spectroscopy (FT-IR)," BIO Web of Conferences, vol. 5, Article ID 02016, 2015.

[18] A. Versari, R. B. Boulton, and G. P. Parpinello, "Effect of spectral pre-processing methods on the evaluation of the color components of red wines using fourier-transform infrared spectrometry," Italian Journal of Food Science, vol. 18, no. 4, pp. 423431, 2006.

[19] L. Laghi, A. Versari, G. P. Parpinello, D. Y. Nakaji, and R. B. Boulton, "FTIR spectroscopy and direct orthogonal signal correction preprocessing applied to selected phenolic compounds 
in red wines," Food Analytical Methods, vol. 4, no. 4, pp. 619-625, 2011.

[20] D. Picque, P. Lieben, P. Chrétien, J. Béguin, and L. Guérin, "Assessment of maturity of loire valley wine grapes by midinfrared spectroscopy," Journal International des Sciences de la Vigne et du Vin, vol. 44, no. 4, pp. 219-229, 2010.

[21] A. Soriano, P. M. Pérez-Juan, A. Vicario, J. M. González, and M. S. Pérez-Coello, "Determination of anthocyanins in red wine using a newly developed method based on Fourier transform infrared spectroscopy," Food Chemistry, vol. 104, no. 3, pp. 12951303, 2007.

[22] Z. Rasines-Perea, N. Prieto-Perea, M. Romera-Fernández, L. A. Berrueta, and B. Gallo, "Fast determination of anthocyanins in red grape musts by Fourier transform mid-infrared spectroscopy and partial least squares regression," European Food Research and Technology, vol. 240, no. 5, pp. 897-908, 2015.

[23] M. Del Alamo-Sanza, J. A. F. Escudero, and R. De Castro Torío, "Changes in phenolic compounds and colour parameters of red wine aged with oak chips and in oak barrels," Food Science and Technology International, vol. 10, no. 4, pp. 233-241, 2004.

[24] Z. Guadalupe and B. Ayestarán, "Changes in the color components and phenolic content of red wines from Vitis vinifera $L$. Cv. "tempranillo" during vinification and aging," European Food Research and Technology, vol. 228, no. 1, pp. 29-38, 2008.

[25] A. Gambuti, R. Capuano, M. T. Lisanti, D. Strollo, and L. Moio, "Effect of aging in new oak, one-year-used oak, chestnut barrels and bottle on color, phenolics and gustative profile of three monovarietal red wines," European Food Research and Techno$\log y$, vol. 231, no. 3, pp. 455-465, 2010.

[26] Y. Glories, "La couleur des vins rouges: 2e. Partie: mesure, origine et interpretation," Connaissance de la Vigne et du Vin, vol. 18, pp. 253-271, 1984.

[27] J. M. McRae, S. Kassara, J. A. Kennedy, E. J. Waters, and P. A. Smith, "Effect of wine $\mathrm{pH}$ and bottle closure on tannins," Journal of Agricultural and Food Chemistry, vol. 61, no. 47, pp. 1161811627, 2013.

[28] S. Kallithraka, Y. Kotseridis, M. Kyraleou et al., "Analytical phenolic composition and sensory assessment of selected rare Greek cultivars after extended bottle ageing," Journal of the Science of Food and Agriculture, vol. 95, no. 8, pp. 1638-1647, 2015.

[29] J. Bakker and C. F. Timberlake, "Isolation, identification, and characterization of new color-stable anthocyanins occurring in some red wines," Journal of Agricultural and Food Chemistry, vol. 45, no. 1, pp. 35-43, 1997.

[30] C. Santos-Buelga, E. M. Frauda-Aricha, S. De Pascual-Teresa, and J. C. Rivas-Gonzalo, "Contribution to the identification of the pigments responsible for the browning of anthocyanin-flavanol solutions," European Food Research and Technology, vol. 209, no. 6, pp. 411-415, 1999.

[31] L. Almela, S. Javaloy, J. Fernández-López, and J. Lôpez-Roca, "Comparison between the tristimulus measurements Yxy and $L^{*} a^{*} b^{*}$ to evaluate the colour of young red wines," Food Chemistry, vol. 53, no. 3, pp. 321-327, 1995.

[32] B. C. Smith, Infrared Spectral Interpretation: A Systematic Approach, CRC Press, 1999.

[33] A. Edelmann, J. Diewok, K. C. Schuster, and B. Lendl, "Rapid method for the discrimination of red wine cultivars based on mid-infrared spectroscopy of phenolic wine extracts," Journal of Agricultural and Food Chemistry, vol. 49, no. 3, pp. 1139-1145, 2001.
[34] F. D. S. Grasel, M. F. Ferrão, and C. R. Wolf, "Development of methodology for identification the nature of the polyphenolic extracts by FTIR associated with multivariate analysis," Spectrochimica Acta Part A: Molecular and Biomolecular Spectroscopy, vol. 153, pp. 94-101, 2016.

[35] A. Ricci, K. J. Olejar, G. P. Parpinello, P. A. Kilmartin, and A. Versari, "Application of Fourier transform infrared (FTIR) spectroscopy in the characterization of tannins," Applied Spectroscopy Reviews, vol. 50, no. 5, pp. 407-442, 2015.

[36] Y. Zhang, J. Chen, Y. Lei, Q. Zhou, S. Sun, and I. Noda, "Discrimination of different red wine by Fourier-transform infrared and two-dimensional infrared correlation spectroscopy," Journal of Molecular Structure, vol. 974, pp. 144-150, 2010.

[37] S. Agatonovic-Kustrin, "The Use of Fourier Transform Infrared (FTIR) spectroscopy and Artificial Neural Networks (ANNs) to assess wine quality," Modern Chemistry \& Applications, vol. 1, pp. 1-8, 2013.

[38] C. P. Passos, S. M. Cardoso, A. S. Barros, C. M. Silva, and M. A. Coimbra, "Application of Fourier transform infrared spectroscopy and orthogonal projections to latent structures/partial least squares regression for estimation of procyanidins average degree of polymerisation," Analytica Chimica Acta, vol. 661, no. 2, pp. 143-149, 2010.

[39] T. E. Coldea, C. Socaciu, F. Fetea, F. Ranga, R. M. Pop, and M. Florea, "Rapid quantitative analysis of ethanol and prediction of methanol content in traditional fruit brandies from romania, using FTIR spectroscopy and chemometrics," Notulae Botanicae Horti Agrobotanici Cluj-Napoca, vol. 41, no. 1, pp. 143-149, 2013. 

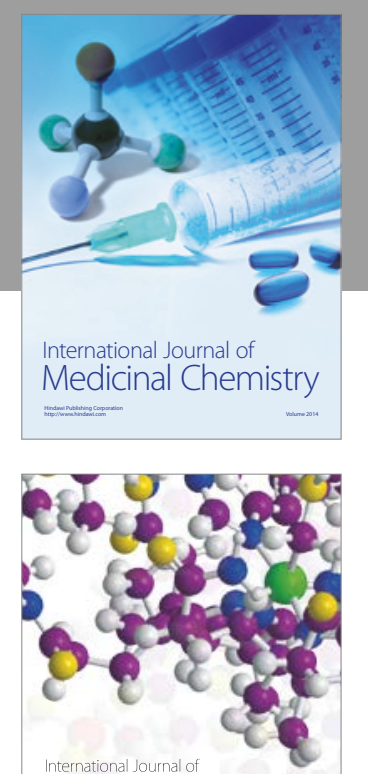

Carbohydrate Chemistry

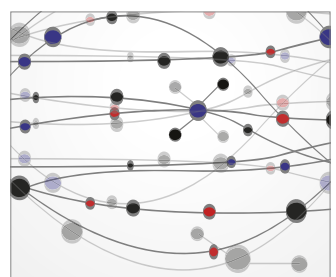

The Scientific World Journal
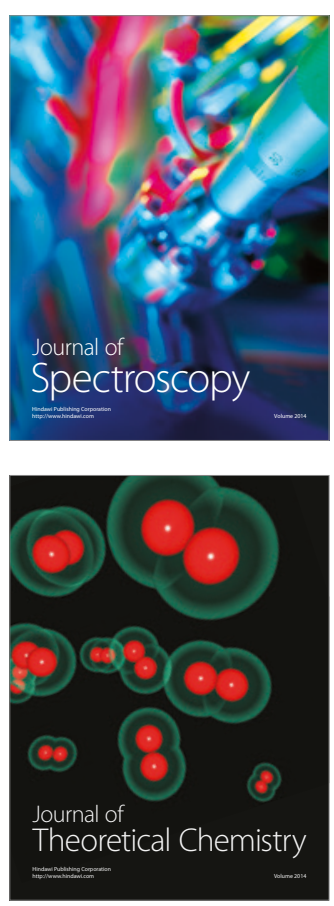
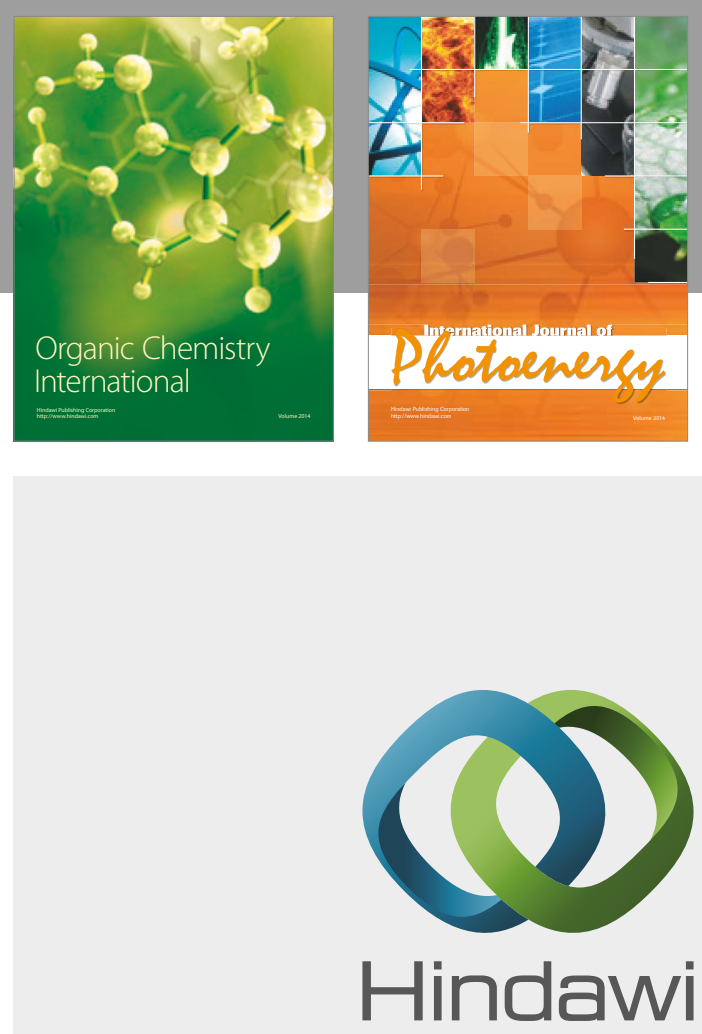

Submit your manuscripts at

https://www.hindawi.com

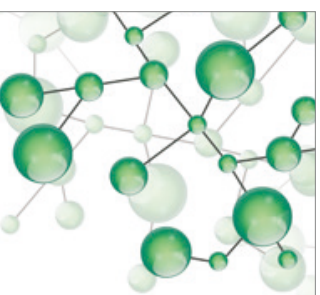

International Journal of

Inorganic Chemistry

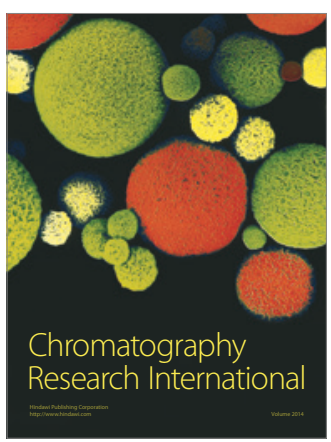

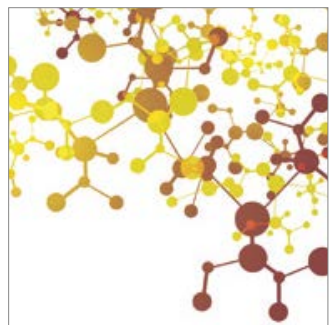

Applied Chemistry
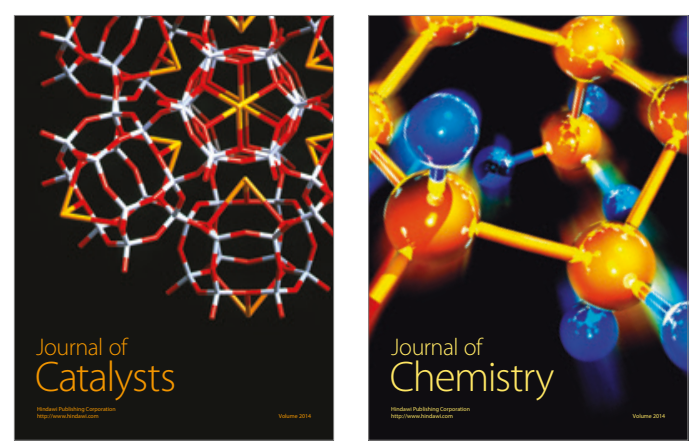
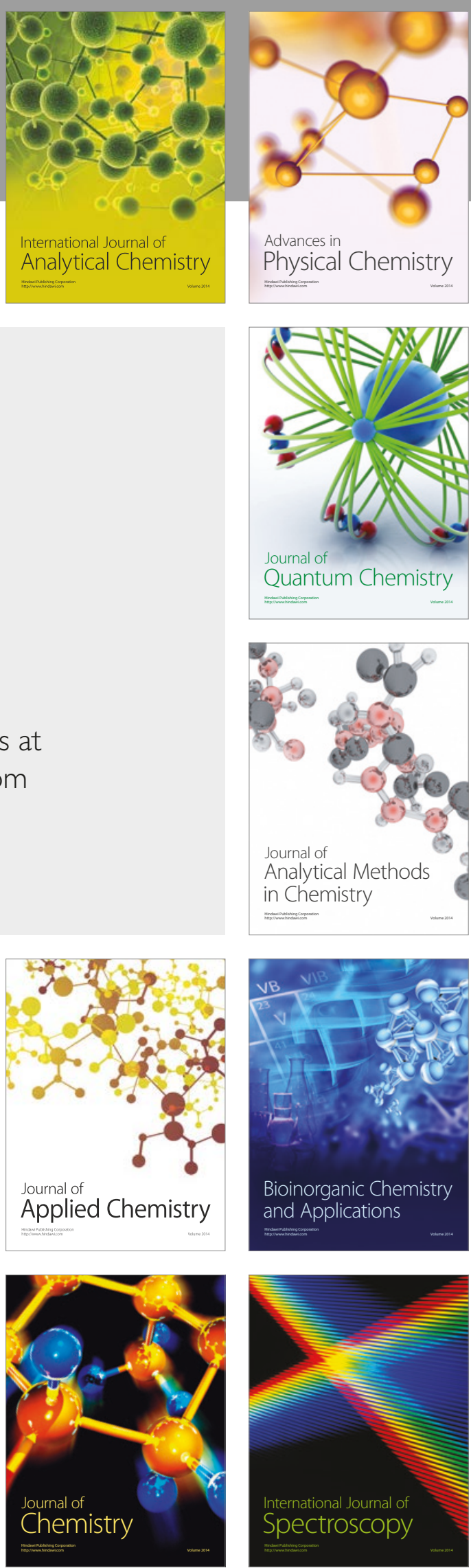